\title{
incidence des conditions d'exécution et du délai de repos sur le comportement et la portance des fondations forées *
}

\author{
par \\ M. Bustamante \\ Laboratoire Central des Ponts et Chaussées \\ D. Gouvenot \\ Solétanche Entreprise
}

\section{Introduction}

Différents auteurs ont montré que la mise en place des pieux modifie les propriétés du sol encaissant, les altérant ou parfois même les améliorant [1], [2], [3].

C'est ainsi que les sables sont densifiés par le battage ou l'injection sous pression, mais décomprimés par le forage ou le lançage. Quant aux sols cohérents, ils sont en règle générale remaniés par la presque totalité des techniques de mise en œuvre (fig. 1).

Sachant, par exemple, que pour les pieux sollicités verticalement l'effet de frottement latéral se concentre au voisinage immédiat du fût [4], on imagine aisément la répercussion du remaniement sur sa portance globale.

Comme on sait par ailleurs que le degré de remaniement peut évoluer dans le temps, on mesure toute la difficulté à laquelle se heurte l'ingénieur lors du dimensionnement du pieu puisque, après la mise en œuvre, il ignore dans quel sens et dans quelle proportion vont évoluer les parametres intervenant dans le calcul.

On tentera d'estimer, à partir d'un certain nombre d'exemples concrets de chargements (ou d'arrachements) en vraie grandeur, comment se répercutent sur la portance, les facteurs:

- mode d'exécution.

- délai de repos.

- incidents en cours de forage.

On démontrera aussi, et en tout premier lieu, qu'indépendamment de l'incidence de ces trois facteurs, les fondations étudiées ont toujours obéi aux mêmes lois générales de comportement.

\section{Identité de comportement de l'ensemble des fondations forées}

La présente étude comparative porte sur un total de 28 fondations profondes (barrette, pieux et tirants), mises en œuvre dans huit types de sols distincts, répartis sur 11 sites différents (fig. 2 ).

\footnotetext{
- Présenté au Comité Français de Mécanique des Sols Séance du
} 9 janvier 1978 .

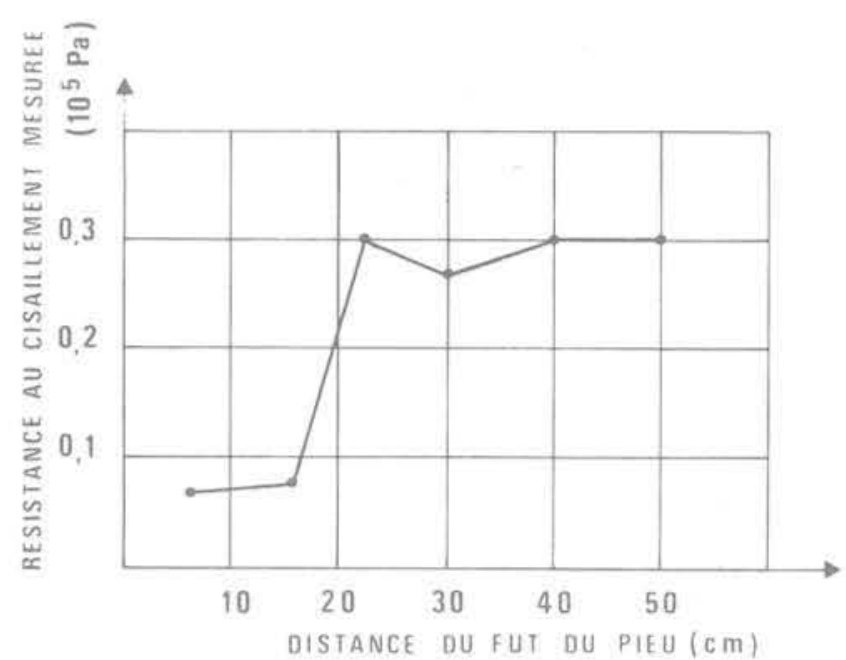

Fig. 1 Résistance au cisaillement au voisinage de la paroi $d^{\prime} u n$ pieu métallique de $900 \mathrm{~mm}$ de diamètre

La totalité de ces fondations a été soumise à des essais de chargement (ou d'arrachement) effectués par Solétanche Entreprise ou les Laboratoires des Ponts-et-Chaussées.

Chaque essai a été poussé jusqu'aux charges (ou tractions) limites (fig. 3). Par souci de clarté, on ne rapportera et commentera que les cas les plus représentatifs.

La comparaison de l'ensemble des courbes et diagrammes caractéristiques :

- droites de stabilisation (fig. 4a (barrette) b (tirant)),

- relations «pente des droites de stabilisation - effort appliqué en tête (fig. 5),

- répartition des déformations unitaires le long de la fondation (fig. 6),

- mobilisations du frottement latéral en fonction du déplacement de la fondation (fig. 7), 
Figure 2: Fondations et tirants étudiés

\begin{tabular}{|c|c|c|c|c|c|c|}
\hline Site & $\begin{array}{c}\text { Type } \\
\text { de } \\
\text { fondation }\end{array}$ & $\begin{array}{c}\text { Mode } \\
\text { de } \\
\text { perforation }\end{array}$ & $\begin{array}{c}\text { Nombre } \\
\text { de } \\
\text { fondations }\end{array}$ & $\begin{array}{c}\text { Nombre d'essais } \\
\text { de chargement } \\
\text { ou d'arrachement }\end{array}$ & Sols & $\begin{array}{l}\text { Appareils de } \\
\text { mesures au sein } \\
\text { de la fondation }\end{array}$ \\
\hline $\begin{array}{c}\text { Vallée de la Risle } \\
1969 \text { à } 1971\end{array}$ & $\begin{array}{c}\text { Pieu béton moulé } \\
\phi 900 \\
\text { Pieu béton moulé } \\
\phi 900 \\
\text { Pieu métallique } \\
\phi 900 \\
\end{array}$ & $\begin{array}{c}\text { Tarière }+ \text { boue } \\
\text { Bénoto } \\
\text { Bénoto }\end{array}$ & $\begin{array}{l}1 \\
1\end{array}$ & $\begin{array}{l}3 \\
2\end{array}$ & $\begin{array}{l}\text { Limons } \\
\text { argileux } \\
\text { (sensibles) }\end{array}$ & $\begin{array}{l}+ \\
+ \\
+\end{array}$ \\
\hline $\begin{array}{c}\text { Nord de la France } \\
1977\end{array}$ & $\begin{array}{c}\text { Pieu béton moulé } \\
\phi 1300 \\
\text { Pieu béton préfab. } \\
\phi 350 \\
\text { Pieu métallique } \\
\phi 180 \\
\end{array}$ & $\begin{array}{c}\text { Tarière à sec } \\
\text { Vérine } \\
\begin{array}{c}\text { Foré coulis tinjection } \\
\text { (sous pression) }\end{array} \\
\end{array}$ & $\begin{array}{l}1 \\
1 \\
1\end{array}$ & $\begin{array}{l}1 \\
1 \\
1\end{array}$ & $\begin{array}{l}\text { Argile }\left(A_{t}\right) \\
\text { des } \\
\text { Flandres }\end{array}$ & $\begin{array}{l}+ \\
+ \\
+\end{array}$ \\
\hline $\begin{array}{c}\text { North Rankin Field } \\
\text { (Australie) } \\
1974\end{array}$ & $\begin{array}{c}\text { Pieu métallique } \\
\phi 340 \\
\text { Pieu métallique } \\
\phi 244\end{array}$ & $\begin{array}{l}\text { Battage } \\
\text { Foré à l'eau claire } \\
\text { et tricone et cimenté } \\
\text { gravitaire }\end{array}$ & $\begin{array}{l}2 \\
2\end{array}$ & 2 & $\begin{array}{l}\text { Sables } \\
\text { calcaires }\end{array}$ & $\begin{array}{l}+ \\
+\end{array}$ \\
\hline $\begin{array}{l}\text { Winnezeele } \\
\quad 1976\end{array}$ & Tirant TMD & $\begin{array}{c}\text { Outil } 0 \text { - Lames } \phi 140 \\
+ \\
\text { coulis ciment }\end{array}$ & 4 & 4 & $\begin{array}{c}\text { Argile }\left(A_{t}\right) \\
\text { des } \\
\text { Flandres }\end{array}$ & + \\
\hline $\begin{array}{l}\text { Douai } \\
1977\end{array}$ & $\begin{array}{l}\text { Tirant à tube } \\
\text { à manchettes } \\
\text { central }\end{array}$ & $\begin{array}{c}\text { Tricone } \phi 140 \\
+ \\
\text { bentonite }\end{array}$ & 3 & 3 & $\begin{array}{c}\text { Argile }\left(A_{t}\right) \\
\text { des } \\
\text { Flandres }\end{array}$ & - \\
\hline $\begin{array}{c}\text { Boulogne } \\
\text { Billancourt } \\
1973\end{array}$ & $\begin{array}{l}\text { Pieu béton moulé } \\
\phi 560 \text { et } 520\end{array}$ & $\begin{array}{c}\text { Tube battu } \\
+ \\
\text { soupape }\end{array}$ & 2 & 2 & $\begin{array}{l}\text { Craie } \\
\text { altérée }\end{array}$ & + \\
\hline $\begin{array}{c}\text { Mantes } \\
1974\end{array}$ & $\begin{array}{c}\text { Pieu béton moulé } \\
\phi 970 \text { à } 690\end{array}$ & $\begin{array}{c}\text { Bucket } \\
+ \\
\text { bentonite } \\
\end{array}$ & 1 & 1 & $\begin{array}{l}\text { Craie } \\
\text { altérée }\end{array}$ & + \\
\hline $\begin{array}{l}\text { ANWB } \\
\text { (La Haye) } \\
1971\end{array}$ & $\begin{array}{c}\text { Pieu béton moulé } \\
\phi 560\end{array}$ & $\begin{array}{c}\text { Tarière } \\
+ \\
\text { bentonite }\end{array}$ & 2 & 2 & $\begin{array}{l}\text { Sables } \\
\text { argileux }\end{array}$ & + \\
\hline $\begin{array}{l}\text { Dunkerque } \\
1973 \text { à } 1974\end{array}$ & $\begin{array}{l}\text { Pieu métallique } \\
\phi 5500\end{array}$ & $\begin{array}{c}\text { Circulation } \\
\text { inverse a l'eau } \\
\phi 660 \\
+ \\
\text { cimentation gravitaire }\end{array}$ & 2 & 2 & $\begin{array}{c}\text { Sables fins } \\
+ \\
\text { vases }\end{array}$ & + \\
\hline $\begin{array}{c}\text { Coudekerque } \\
1973\end{array}$ & $\begin{array}{l}\text { Pieu façonné } \\
(35 \times 35) \mathrm{cm}\end{array}$ & $\begin{array}{c}\text { Battage } \\
+ \\
\text { lancage }\end{array}$ & 3 & 3 & $\begin{array}{l}\text { Sables } \\
\text { moyens } \\
\text { compacts }\end{array}$ & + \\
\hline $\begin{array}{c}\text { St Nazaire } \\
1977\end{array}$ & $\begin{array}{c}\text { Barrette } \\
(2,20 \times 0,62) \mathrm{m}\end{array}$ & $\begin{array}{c}\text { Kelly } \\
+ \\
\text { bentonite }\end{array}$ & 1 & 1 & $\begin{array}{l}\text { Vases } \\
\text { et } \\
\text { gneiss }\end{array}$ & + \\
\hline \multicolumn{3}{|c|}{ TOTAL } & 28 & 33 & & - \\
\hline
\end{tabular}

+ Fondation équipée d'extensomètres

- Fondation ne comportant pas d'extensomètre

Fig. 2 Caractéristiques des différents types de fondations profondes étudiées 


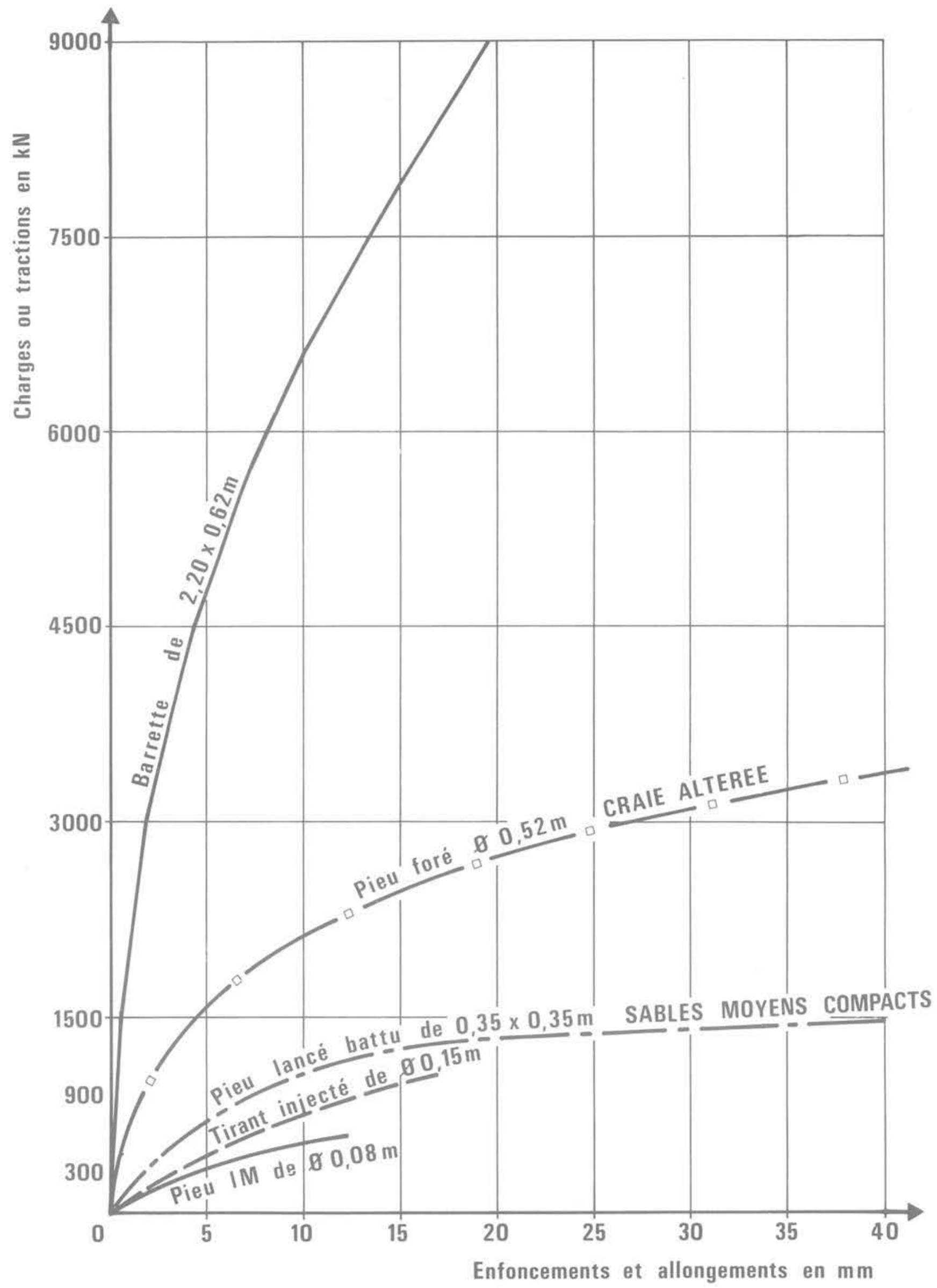

Fig. 3 Résultats d'essais de chargement ou d'arrachement sur des fondations très diverses 
Temps en mn.

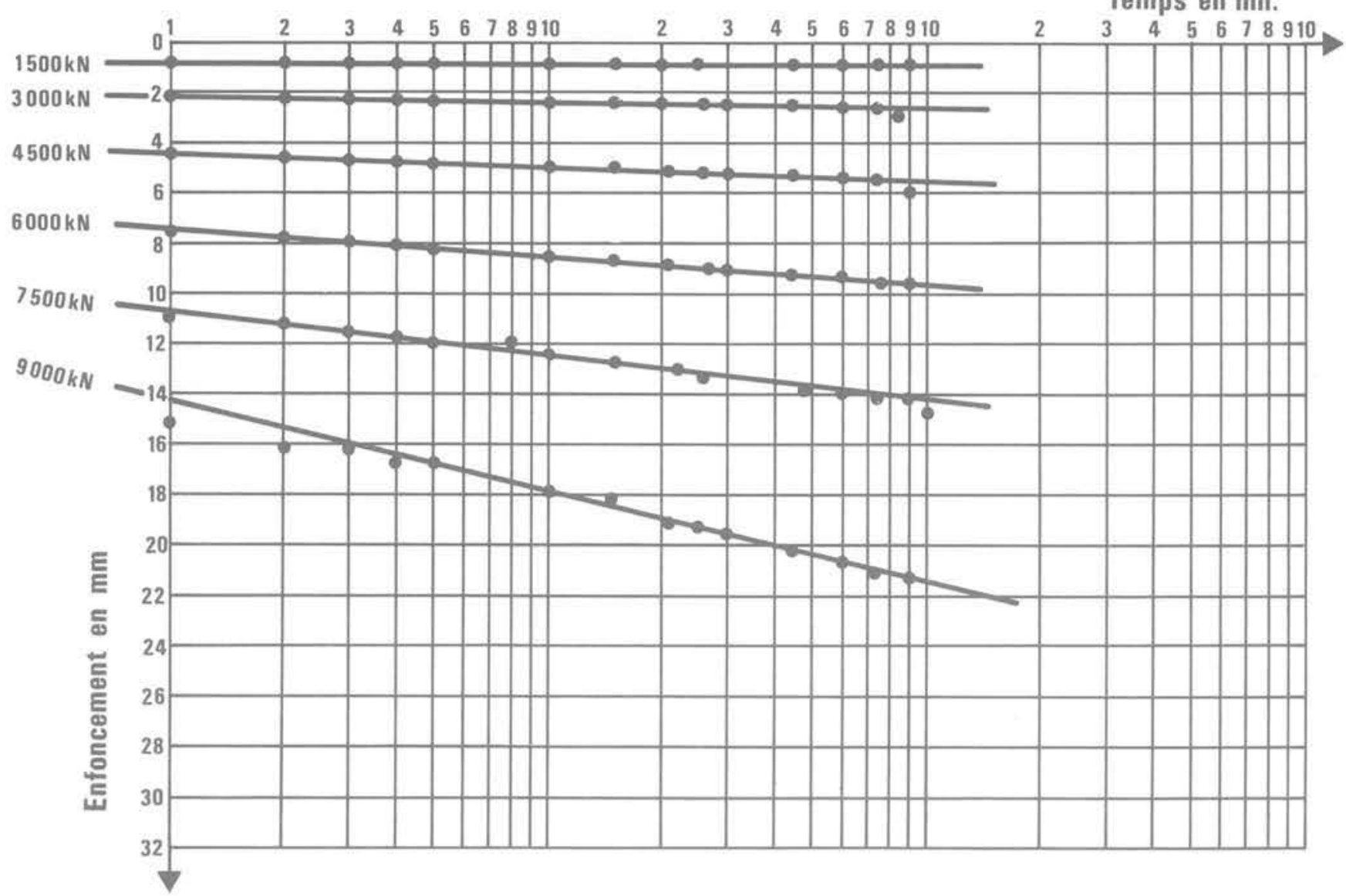

4 a Barrette de $2,20 \times 0,60 \mathrm{~m}$

$\log 1(\mathrm{mn})$

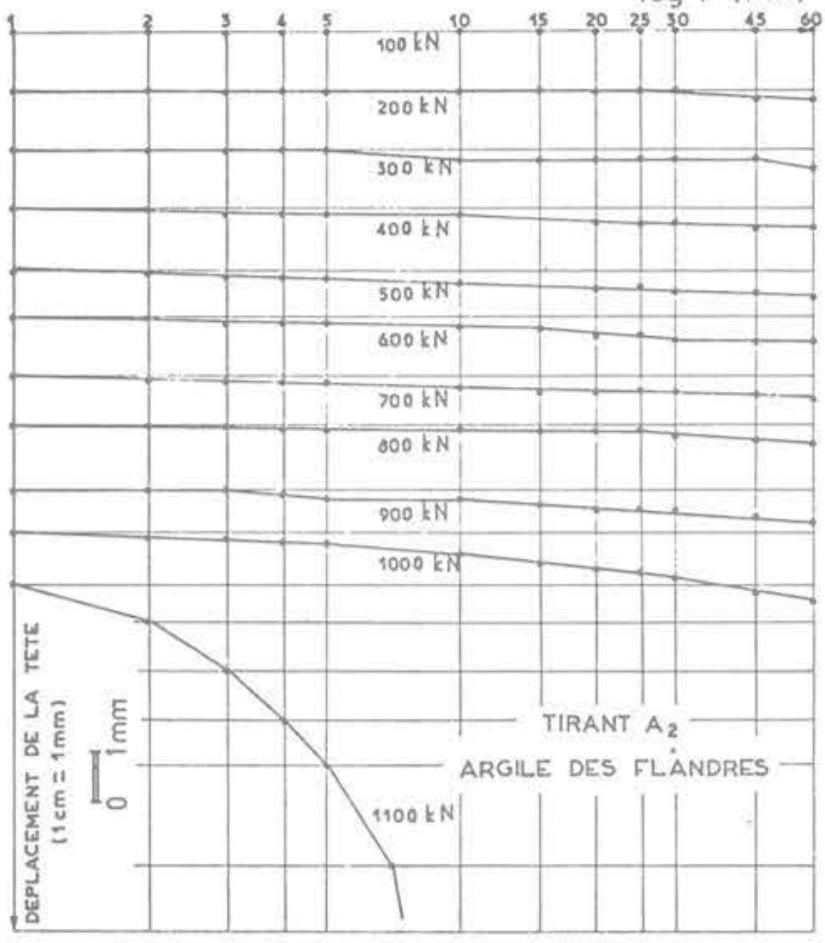

$4 \mathrm{~b}$ Tirant de $150 \mathrm{~mm}$ de diamètre ancré dans l'argile des Flandres 


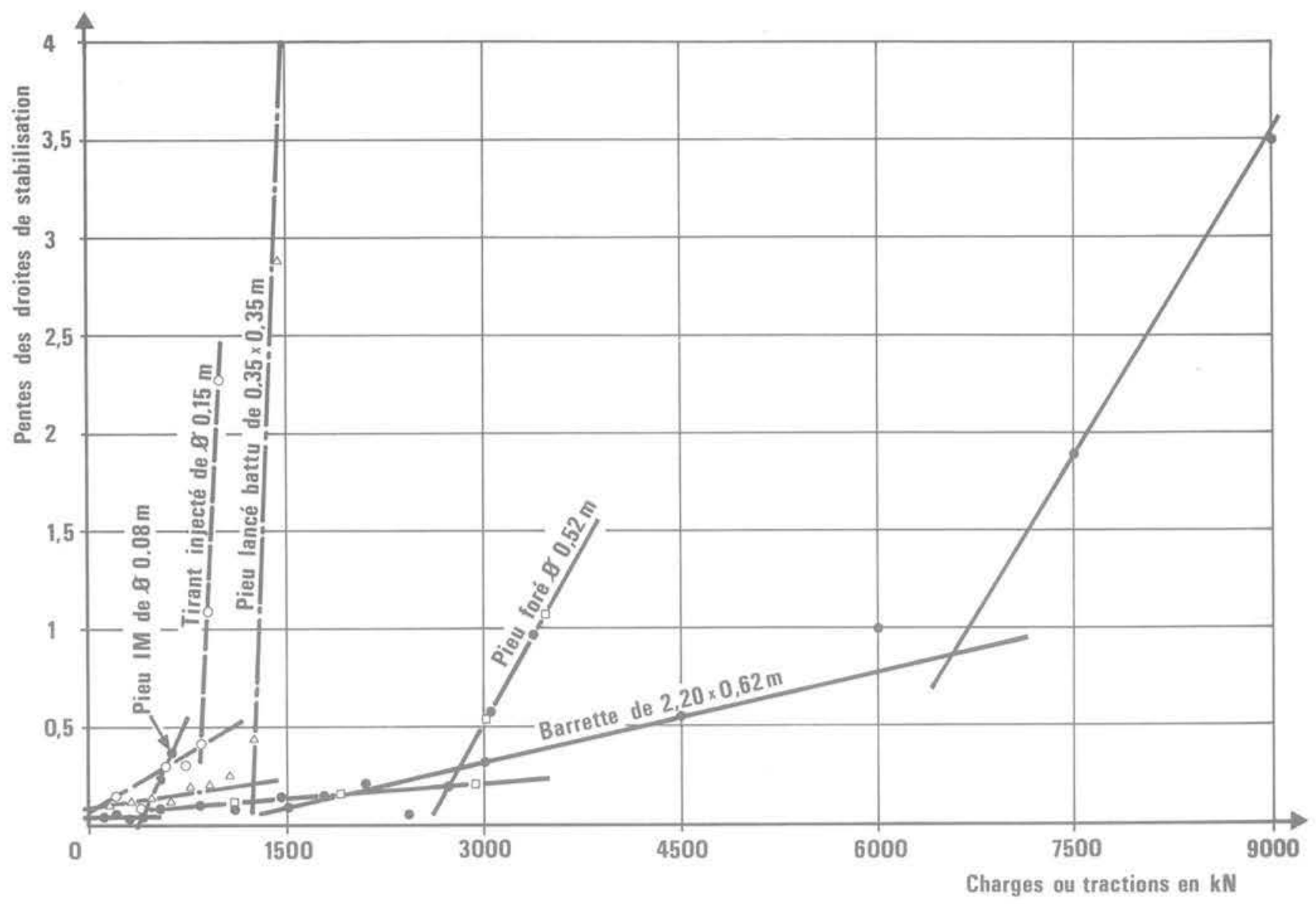

Fig. 5 Exemples de variations des pentes des droites de stabilisation en fonction des efforts

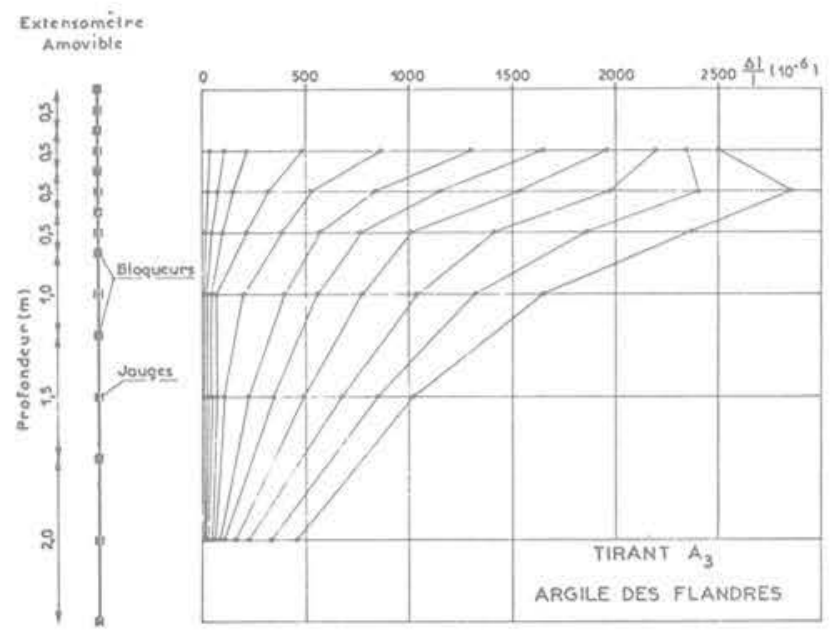

Fig. 6 Répartition des déformations unitaires le long d'un tirant 


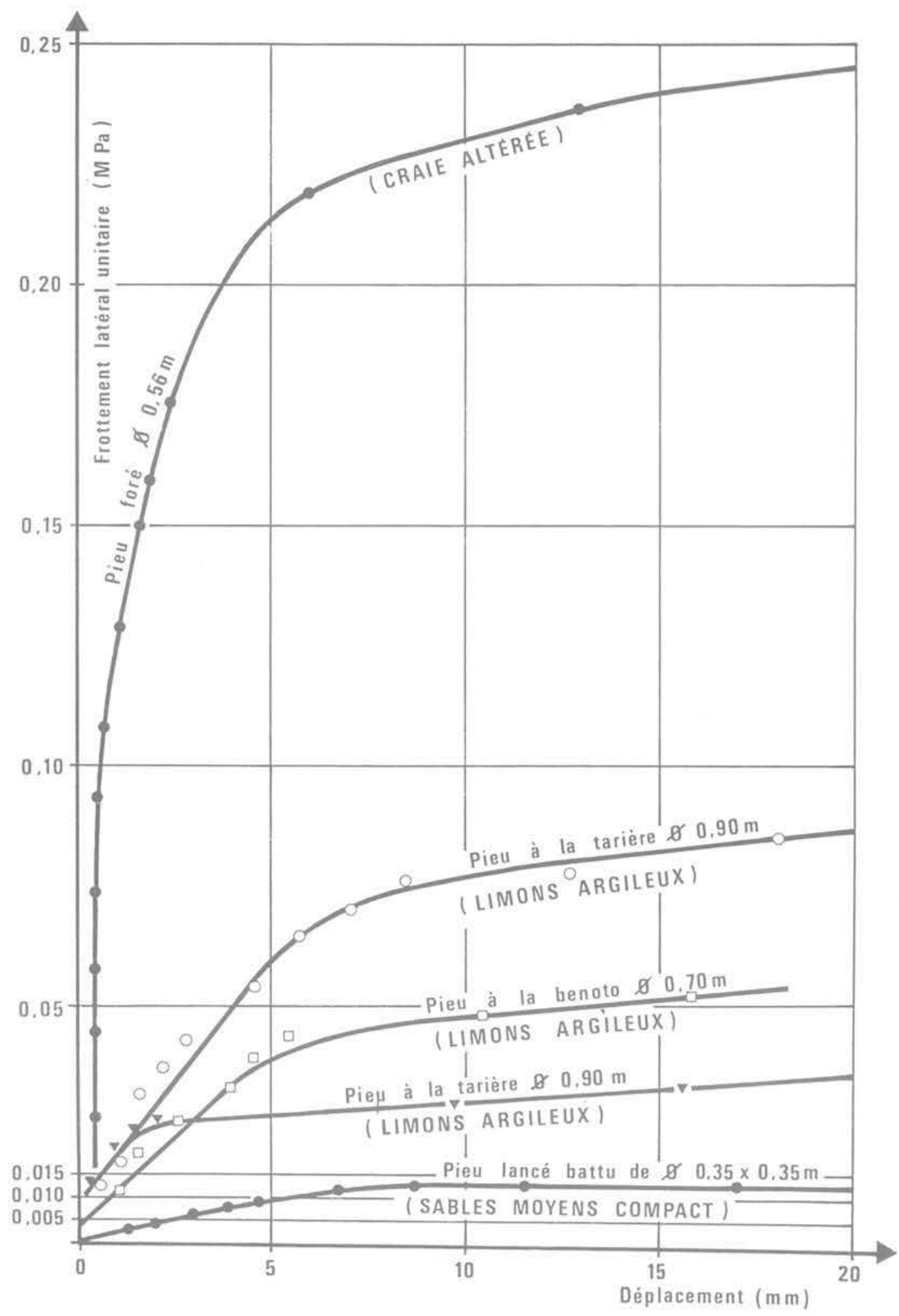

Fig. 7 Mobilisation du frottement latéral en fonction du déplacement de la fondation pour différents types de pieux 
confirme, par la similitude des allures, l'identité de comportement mais aussi le fait que toutes ces fondations obéissent aux lois de comportement retenant le principe d'une mobilisation progressive (ou simultanée) de la résistance de pointe et du frottement latéral [5], [6] (fig. 8).

C'est d'ailleurs cette identité de comportement qui a finalement conduit à retenir comme méthode d'interprétation des essais de chargement (ou d'arrachement) les mêmes critères pour tous les types de fondations profondes [7], [8].

\section{Incidence du mode d'exécution}

Celle-ci, pour un sol donné, se résume à 2 cas :

- à différents modes d'exécution correspondent des capacités portantes (ou d'ancrage) différentes (tableaux 1, 2 , 3 et 4$)$.

- à différents modes d'exécution peuvent également correspondre des capacités portantes (ou d'ancrage) identiques ou très voisines (tableau 5 ).

\section{Cas du tableau 1}

Ce tableau présente les valeurs des frottements latéraux unitaires $\left(f_{1 \text { at }}\right)$ obtenues pour 3 pieux expérimentaux soumis sur une période de 3 ans, à plusieurs essais de chargements statiques [9].

Ces trois pieux, de $20 \mathrm{~m}$ de longueur et de $900 \mathrm{~mm}$ de diamètre, sont encastrés de $10 \mathrm{~m}$ dans un limon argileux peu à très plastique de l'albo-aptien. Une analyse diffractométrique a mis en évidence la teneur en montmorillonite importante de ces limons et, par conséquent, leur aptitude au remaniement. Au triaxial, l'ensemble des essais $a$ indiqué une valeur moyenne de la cohésion $C_{u}=0,15$ $\mathrm{MPa}$.

Les pieux consistaient en :

- un pieu réalisé à la tarière sous boue bentonitique.

- un pieu foré à la Benoto sous boue bentonitique.

- un pieu métallique, louvoyé par Benoto et fermé à la base.

Pour les deux premiers pieux en béton, la comparaison des valeurs de frottement $\left(f_{\text {lat }}\right.$ ) montre que, dans le cas précis de ce limon, l'exécution d'un forage à la tarière sous boue

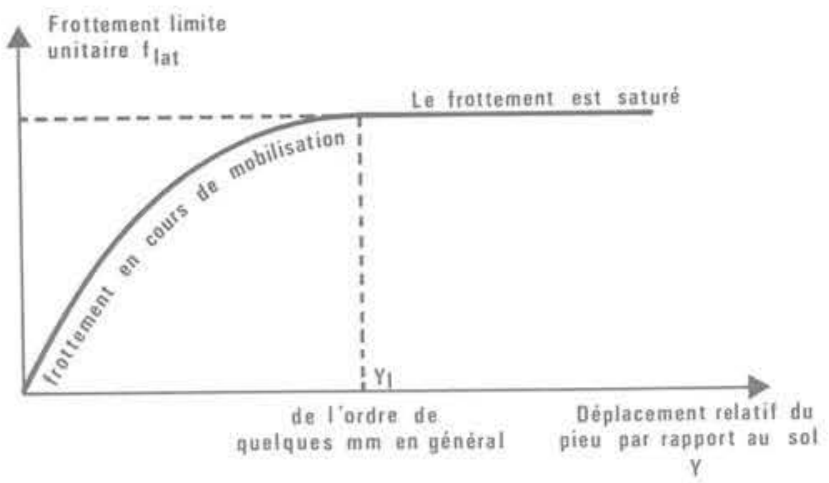

Fig. 8 Lai générale de frottement traduisant la mobilisation progressive du frottement latéral le long du fat d'un pieu

remanie moins le sol autour du fât que la Benoto. Le fût métallique du $3^{\circ}$ pieu, mis également en œuvre par Benoto, présente une valeur encore plus faible du frottement.

Dans tous les cas, on ne peut que constater l'importance du remaniement: pour les premiers essais, la valeur de $f_{\text {lat }}$ ne constitue respectivement que 35,30 et $14 \%$ de la résistance $C_{u}$ initiale $(0,15 \mathrm{MPa})$. On verra par la suite comment évoluèrent ces mêmes valeurs dans le temps. L'analyse des autres résultats présente le même intérêt. Ainsi, les charges limites sous la pointe $\left(Q_{p}^{L}\right)$ valaient dans le même ordre: 1300,1350 et $1150 \mathrm{KN}$ pour les premiers chargements. En raison de l'identité des sections pour les trois pieux, ces dernières valeurs, d'ailleurs très proches, confirment le remaniement extrême du sol sous les pointes.

Ces mêmes termes de pointes $\left(Q_{p}^{L}\right)$ sont à comparer aux valeurs calculées à partir du pressiomètre $(2000 \mathrm{kN})$, du pénétromètre statique $(2500 \mathrm{kN})$ et des essais de laboratoire ou du scissomètre $(900 \mathrm{kN})$. Cette dernière valeur incite à penser que le taux de remaniement sous la pointe des pieux s'est trouvé être du même ordre que le remaniement du sol lors de son prélèvement.

Tableau 1 Viaduc de la Risle (1969 à 1971)

Types de fondations:

1. Pieu béton moulé foré à la tarière sans boue $\emptyset 900 \mathrm{~mm}$

2. Pieu béton foré à la Bénoto $\emptyset 900 \mathrm{~mm}$

3. Pieu métallique $\emptyset 880 \mathrm{~mm}$ mis en place par machine Bénoto

Profil géotechnique

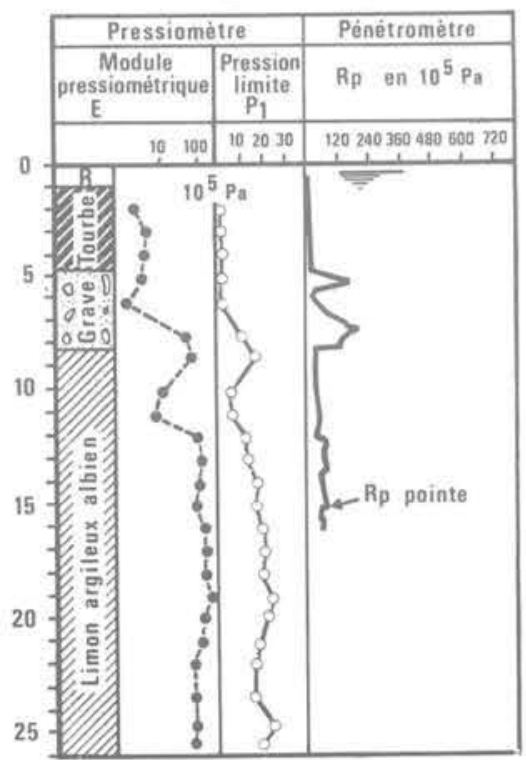

Fiche des pieux

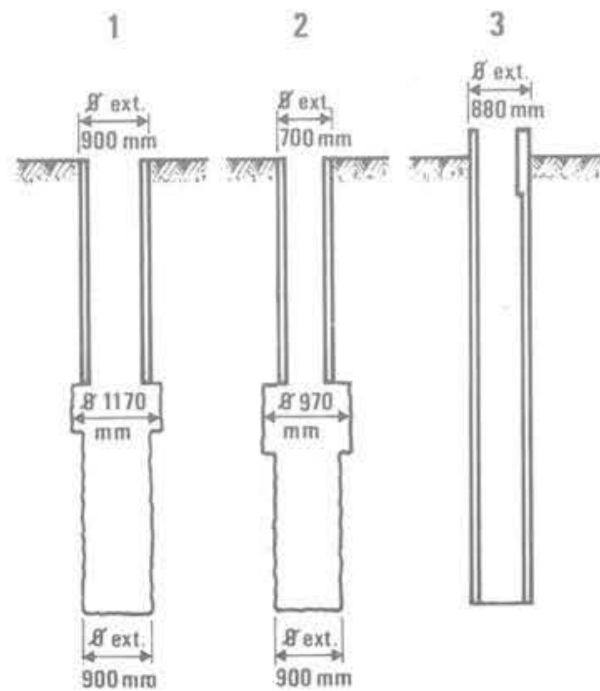

Frottement latéral au $1^{\text {er }}$ essai 86 à 150 jours après la mise en œuvre

\begin{tabular}{|c|c|c|c|}
\hline Pieu $N^{0}$ & 1 & 2 & 3 \\
\hline$f_{\text {lat en } 10^{5} \mathrm{~Pa}}$ & 0,52 & 0,46 & 0,22 \\
\hline Rapport 1/2 & 1,13 & - & - \\
\hline Rapport $1 / 3$ & 2,36 & - & - \\
\hline
\end{tabular}




\section{Cas du tableau 2}

Suite à différents essais réalisés dans une argile de 0,08 à $0,12 \mathrm{MPa}$ de cohésion non drainée [10], on a pu étudier, dans le but de dimensionner différents types de pieux, les variations du rapport frottement limite/cohésion non drainée du sol, rapport appelé $\alpha$ et qui traduit en fait le taux de remaniement du sol:

$$
\alpha=\frac{f_{\text {lat }}}{C_{u}}
$$

Pour différents types de pieux forés sans boue à la tarière en $1300 \mathrm{~mm}$ de diamètre, le remaniement du sol a été considérable puisque le coefficient $\alpha$ a pu être estimé à $0,1-0,15$.

Par contre, pour un pieu vériné de $350 \mathrm{~mm}$ de diamètre, $\alpha$ a atteint $0,4-0,5$, valeur très correcte et semblant même constituer un maximum si l'on se réfère à différents auteurs [11]. [12].

Toutefois, des pieux métalliques de $130 \mathrm{~mm}$ de diamètre forés au coulis de ciment puis scellés par gravité dans leur forage $(\varnothing 180 \mathrm{~mm})$ au coulis fortement dosé $(C / E=2,4)$, ont permis d'atteindre des coefficients $\alpha$ supérieurs à 0,7 .

Ces différentes expériences montrent nettement l'influence du mode d'exécution. Quant aux bonnes performances des pieux métalliques, elles semblent pouvoir être attribuées d'une part au faible diamètre de perforation $(\varnothing 180 \mathrm{~mm})$ qui décomprime probablement moins le sol qu'un forage à sec en $\varnothing 1300 \mathrm{~mm}$, d'autre part à l'utilisation d'une boue de forage au ciment dont l'effet bénéfique est reconnu sur les argiles [10], II s'ensuit que les pieux métalliques scellés de ce type présentent des capacités bien supérieures à ce que laissaient prévoir les méthodes de calcul habituelles. Le pieu vériné, qui provoque le refoulement du sol. bénéficie certainement d'un bon contact du sol, le long de son fât, mais l'adhérence "béton préfabriqué-sol argileux" n'est guère meilleure que celle du contact «acier-sol argileux $》$.

- Le rapport $C / E$, qui caractérise un coulis, exprime le rapport des poids du ciment et de l'eau entrant dans la composition de ce coulis. Ainsi, un rapport $\mathrm{C} / \mathrm{E}$ égal à 2,4 indique un coulis obtenu en malaxant $150 \mathrm{~kg}$ de ciment et environ 62 litres d'eau.

\section{Tableau 2 Argile plastique ou très plastique}

\begin{tabular}{|c|c|c|}
\hline $\begin{array}{l}\text { Coefficient de } \\
\text { frottement latéral }\end{array}$ & & $=\frac{\text { flat }}{C_{u}}=$ \\
\hline $\begin{array}{l}\text { Pieu à la tarière } \\
\text { à sec } \phi 1300 \mathrm{~mm}\end{array}$ & $\alpha$ & de 0,1 à \\
\hline $\begin{array}{l}\text { Pieu en béton } \\
\text { préfabriqué } \\
\text { vériné } \phi 350 \mathrm{~mm}\end{array}$ & $\alpha$ & de 0,4 a \\
\hline $\begin{array}{l}\text { Pieu métallique } \\
\text { foré au coulis et } \\
\text { scellé par gravité }\end{array}$ & $\alpha$ & $>0,7$ \\
\hline
\end{tabular}

\section{Cas du tableau 3}

Sur un champ pétrolier situé au large des côtes australiennes (North Rankin Fieid) [13], on a été amené à battre des pieux métalliques de $340 \mathrm{~mm}$ de diamètre pour fonder une plateforme de forage.

Le sol était constitué d'un sable calcaire dont les grains étaient cimentés à des degrés variables.

Le battage détruisant la structure cimentée du sable, il réduisit considérablement le frottement le long des fûts des pieux. C'est bien ce que montrèrent des essais d'arrachement effectués sur ces pieux: le frottement limite maximum n'atteignant que $30 \mathrm{kPa}$ à $40 \mathrm{~m}$ et $75 \mathrm{~m}$ de profondeur sous le niveau de la mer.

Devant d'aussi faibles valeurs, il fut décidé de tester, sur ce même site, des pieux métalliques de $250 \mathrm{~mm}$ de diamètre mis en place dans un forage de $480 \mathrm{~mm}$ réalisé au tricône et à l'eau claire. Les pieux métalliques furent ensuite scellés au sol par injection gravitaire d'un coulis de ciment $(C / E \simeq 2,4)$. L'interprétation des essais d'arrachement réalisés sur ces derniers pieux indiqua des valeurs du frottement limite maximum supérieures à $80 \mathrm{kPa}$ soit plus de deux fois supérieures à celles mesurées sur des pieux simplement battus.

Cet exemple illustre plusieurs faits :

- le battage, considéré souvent coinme efficace en raison du refoulement du terrain, ne conduit pas nécessairement aux meilleurs résultats. Dans le cas d'un sol à rupture fragile, comme celui du North Rankin Field, sa structure est

Tableau 3 North Rankin Field (Australie) (1974)

Type de fondations : $\emptyset 340$ et $244 \mathrm{~mm}$

Perforation : 2 pieux battus $\emptyset 340 \mathrm{~mm}-2$ pieux forés à l'eau claire au tricône $\emptyset 48,3 \mathrm{~cm}$ et cimentés

Géotechnique du site :

- Sables calcaires dont les grains sont cimentès

\section{- Structure fragile}

- Site offshore: $100 \mathrm{~m}$ d'eau environ
Fiche des pieux

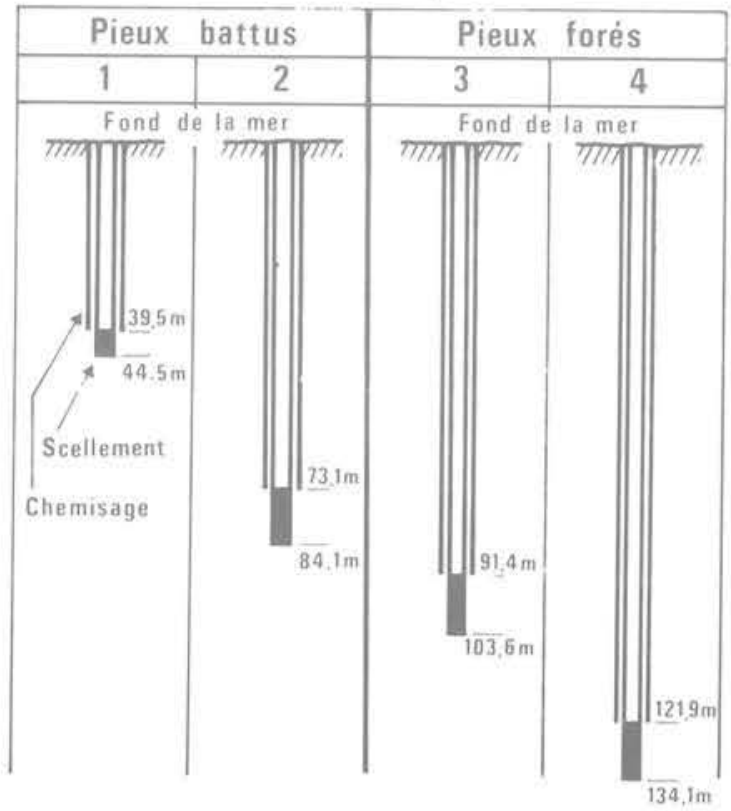

Frottement latéral f lat en $10^{5} \mathrm{~Pa}$

\begin{tabular}{|c|c|c|}
\hline Pieu & Pic & Limite \\
\hline 1 & - & 0,3 \\
\hline 2 & 0,34 & 0.2 \\
\hline 3 & $>0,83$ & - \\
\hline 4 & $>0,98$ & - \\
\hline \multicolumn{2}{|c|}{ Rapport $\frac{3 \text { et } 4}{1 \text { et } 2} \sim 3$} \\
\hline
\end{tabular}


détruite par battage et il n'y a plus d'effet de serrage du sol sur le fût du pieu.

- l'état de surface du pieu vis-à-vis du sol joue un rôle essentiel. Dans le cas du pieu battu métallique, le fût est lisse et le frottement atteint est environ 3 fois plus faible que pour le même pieu, scellé au coulis de ciment, par gravité. Le remplissage de l'espace annulaire entre le pieu métallique et la paroi du forage par du coulis réalise une imbrication intime entre le fat du pieu et le sol. Avec une étreinte latérale faible, la rugosité du fût du pieu conduit malgré tout à un frottement notable $(80$ à $100 \mathrm{kPa})$.

- enfin, qu'une injection de coulis sous pression élevée devrait pouvoir encore augmenter et l'étreinte latérale et l'effet de rugosité : la résistance du sol en place se trouvant ainsi mieux utilisée.

\section{Cas du tableau 4}

$\mathrm{Ce}$ tableau ne rapporte que les valeurs des tractions limites $\left(T_{1}\right)$ obtenues lors d'essais de tirants [14], scellés dans une argile des Fiandres. Les quantités de ciment pour la réalisation des scellements ont été du même ordre $(1500 \mathrm{~kg})$ pour chaque tirant. Chaque série a été injectée en 3 phases, et cela au double obturateur. Seules les techniques de perforation ont été différentes:

- outil à lames + coulis de ciment pour la série des 4 premiers tirants.

- tricône + bentonite pour la série des 3 derniers tirants. Cette différence peut expliquer le rapport de 1 à 1,6 entre la traction limite $\left(T_{L}\right)$ moyenne des trois derniers tirants et celle des quatre premiers.

$$
\text { ARGILE DES FLANDRES }\left(A_{t}\right): \quad \begin{aligned}
0,6 & <p_{l}<0,9 \mathrm{MPa} \\
& 1<\mathrm{Rp}_{\mathrm{p}}<1,5 \mathrm{MPa}
\end{aligned}
$$

DIAMETRE DU FORAGE : $140 \mathrm{~mm}$

INJECTION: SOUS PRESSION + DOUBLE OBTURATEUR

QUANTITE DE COULIS PAR TIRANT: $1500 \mathrm{~kg}$. CIMENT

\begin{tabular}{|c|c|c|}
\hline TECHNIQUE DE FORAGE & $\begin{array}{c}\text { OUTIL A LAMES } \\
+\begin{array}{c}\text { TRICONE } \\
+ \\
\text { COULIS CIMENT }\end{array}\end{array}$ & $\begin{array}{c}+ \\
\text { BENTONITE }\end{array}$ \\
\hline NOMBRE D'ESSAIS & 4 & 3 \\
\hline $\begin{array}{c}\text { TRACTIONS LIMITE TL } \\
(\text { KN })\end{array}$ & $\begin{array}{c}1000 \dot{\mathrm{j}} 1200 \\
(1125)^{*}\end{array}$ & $\begin{array}{c}670 \stackrel{2}{2} 740 \\
(710)^{*}\end{array}$ \\
\hline RAPPORT DE TRACTIONS & \multicolumn{2}{|c|}{$1,6: 1$} \\
\hline
\end{tabular}

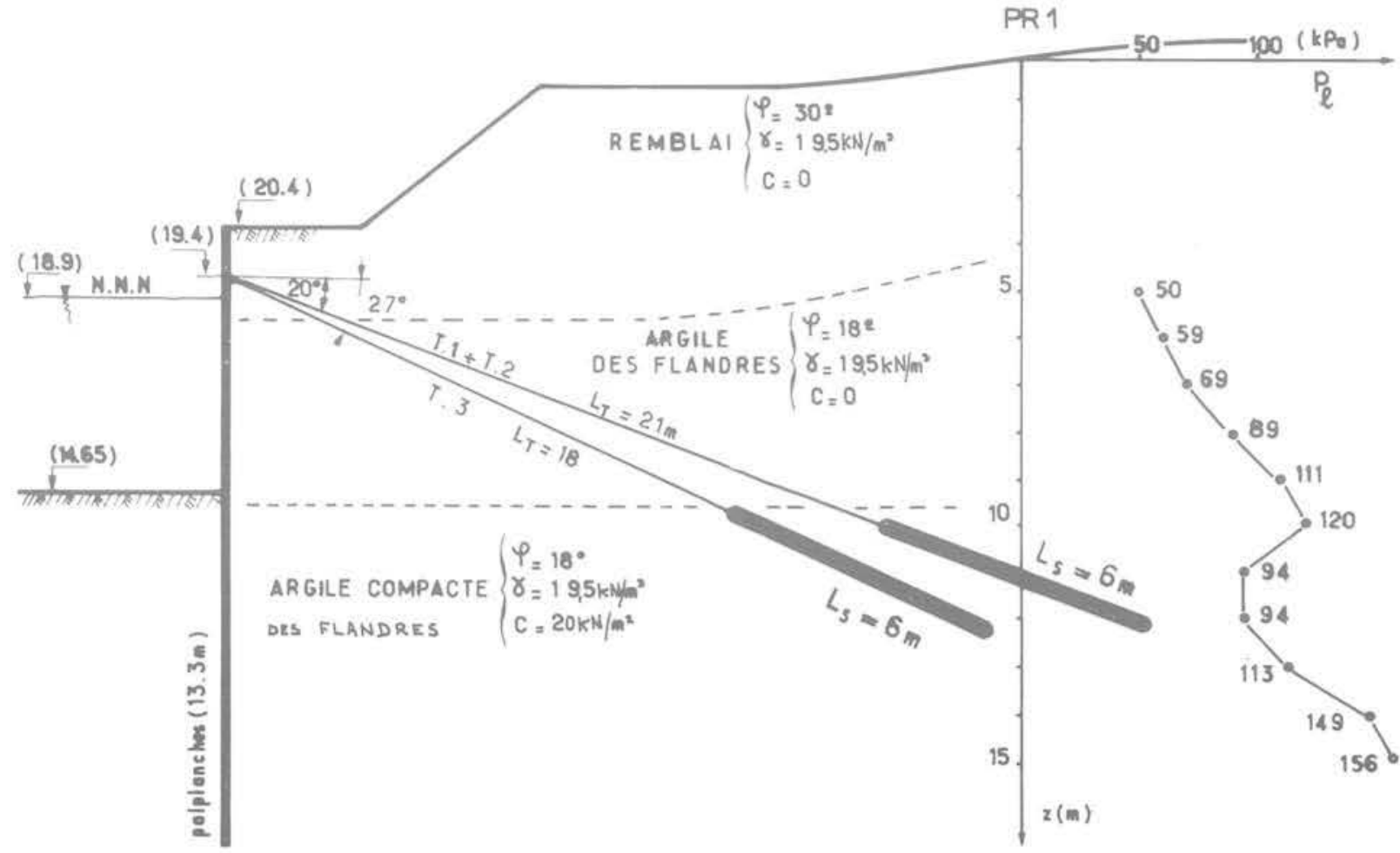


Cas du tableau 5

Celui-ci présente les valeurs de frottement latéral unitaire $\left(f_{\text {lat }}\right)$ obtenues pour 3 pieux forés dans la craie altérée de la Région Parisienne [15], [16]. Les deux premiers pieux (Boulogne-Billancourt) ont été forés à l'eau suivant le procédé classique qui consiste à battre un tubage métallique et à extraire au fur et à mesure de sa pénétration les matériaux à la soupape. Le $3^{\circ}$ pieu (Mantes), pour sa partie fichée dans la craie, a été foré au "bucket " sous boue bentonitique. Dans les deux cas, les pieux ont été bétonnés au tube plongeur.

L'interprétation des mesures a permis de constater que, pour une craie altérée de même capacité $(1,5<\mathrm{PI}$ $<2,0 \mathrm{MPa}$ ) des techniques de perforation utilisées, aucune ne s'est avérée être plus défavorable que l'autre: les frottements latéraux $\left(\mathrm{f}_{\text {lat }}\right)$ moyens étant du même ordre.

\section{Répercussion d'un incident en cours de perforation}

On peut illustrer ce cas par deux exemples caractéristiques, ceux des tableaux 6 et 7 .

Cas du tableau 6

A La Haye en 1971, ont été forés deux pieux en béton moulé de $560 \mathrm{~mm}$ de diamètre. Sur un des deux pieux, l'outil de forage a été cassé et est tombé au fond du forage. Vingt-quatre heures de manœuvres ont été nécessaires pour repêcher l'outil. Outre la décompression du terrain qui a pu se développer pendant ce laps de temps, les différentes opérations de repêchage ont pu remanier la paroi du forage.

Le frottement limite $\left(f_{\text {lat }}\right)$ a atteint la moitié du frottement obtenu sur l'autre pieu dont le forage n'est resté ouvert que les quelques heures riécessaires à l'exécution du bétonnage.

\section{Cas du tableau 7}

A Dunkerque, on a pu effectuer en 1974 une série d'essais de chargement sur des pieux métalliques scellés au sol par injection gravitaire d'abord, puis sous pression élevée ensuite [17]. On n'évoquera ici que le cas des pieux simplement scellés par injection gravitaire.

Ces pieux furent forés avec circulation inverse, à l'eau claire. Pour le premier pieu A1, toutes les opérations se déroulèrent normalement et le frottement limite $\left(f_{\text {lat }}\right)$ atteignit $77 \mathrm{kPa}$ aे l'arrachement.

Pour le pieu A3, le forage s'éboula de nombreuses fois et il dut finalement être foré sous la boue, après deux jours de tentatives infructueuses. La décompression des parois du forage s'est répercutée sur la tenue du pieu à l'arrachement, comme en témoigne la valeur du frottement limite valant finalement $36 \mathrm{kPa}$, soit environ deux fois moins que celle obtenue pour le pieu A1 $(70 \mathrm{kPa})$ dont le forage s'était déroulé sans incidents.

Tableau 5

\begin{tabular}{|c|c|c|}
\hline PIEU & $\begin{array}{c}\text { DIAME TRE DANS } \\
\text { LA CRAIE }\end{array}$ & $\begin{array}{c}\text { FICHE DANS LA CRAIE } \\
(\mathrm{m})\end{array}$ \\
\hline 1 & 0,56 & 4,75 \\
\hline 2 & 0,52 & 7,50 \\
\hline \multicolumn{2}{|c|}{ FORAGE : TUBE BATTU + SOUPAPE } \\
\hline
\end{tabular}

\begin{tabular}{|c|c|c|}
\hline PIEU & $\begin{array}{c}\text { DIAMETRE DANS } \\
\text { LA CRAIE ( } \mathrm{m})\end{array}$ & $\begin{array}{c}\text { FICHE DANS LA CRAIE } \\
(\mathrm{m})\end{array}$ \\
\hline 1 & $0,97 \overline{0} 0,69$ & 9,25 \\
\hline \multicolumn{3}{|c|}{ FORAGE : BUCKET + BENTONITE } \\
\hline
\end{tabular}

\section{PROFIL GEOTECHNIQUE}

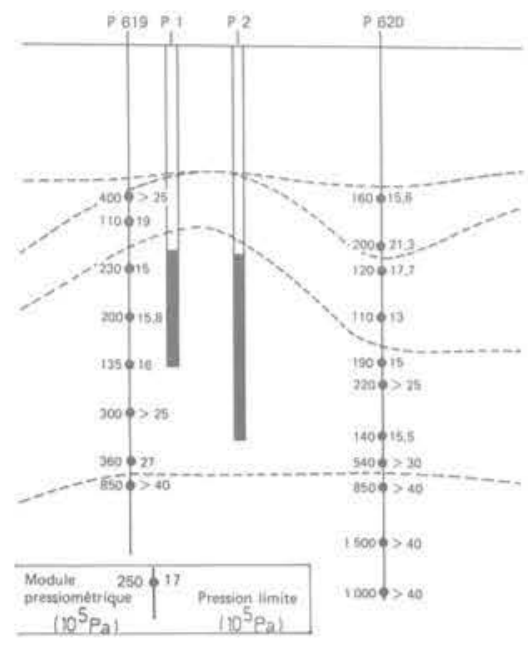

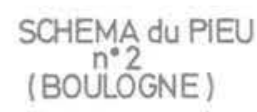

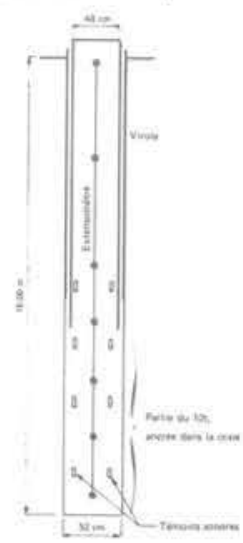

MOBILISATION dU FROT TEMENT LATERAL

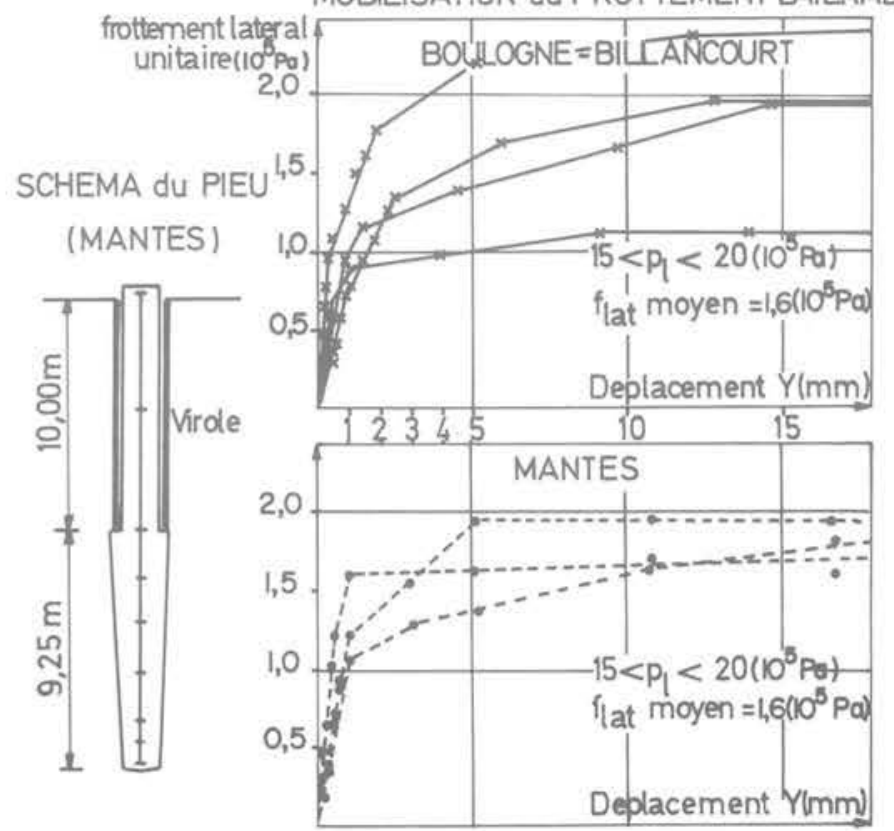


Tableau 6 ANWB - La Haye (1971)

Type de fondations : pieux béton moulés

Géométrie : $\emptyset 560 \mathrm{~mm}$

Perforation : tarière avec boue bentonitique
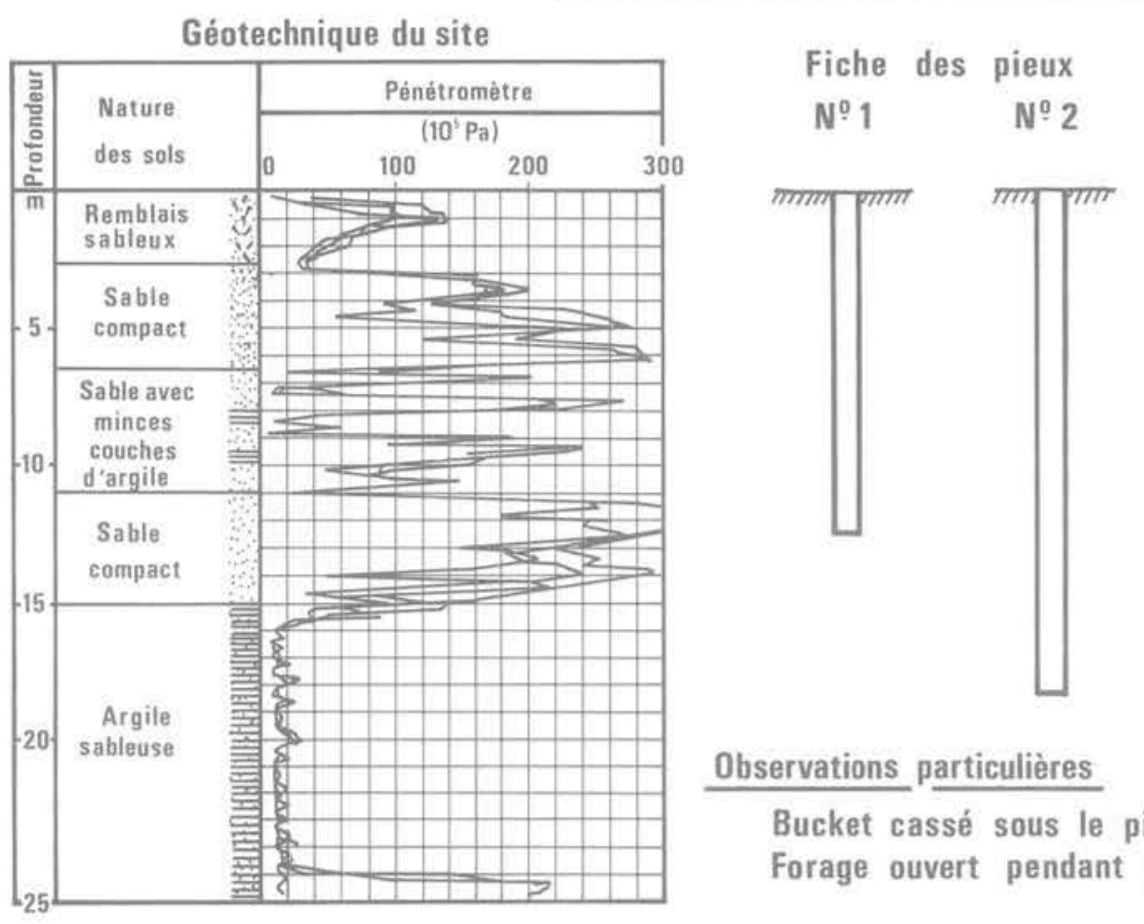

Froţement latéral en $10^{5} \mathrm{~Pa}$

Limite

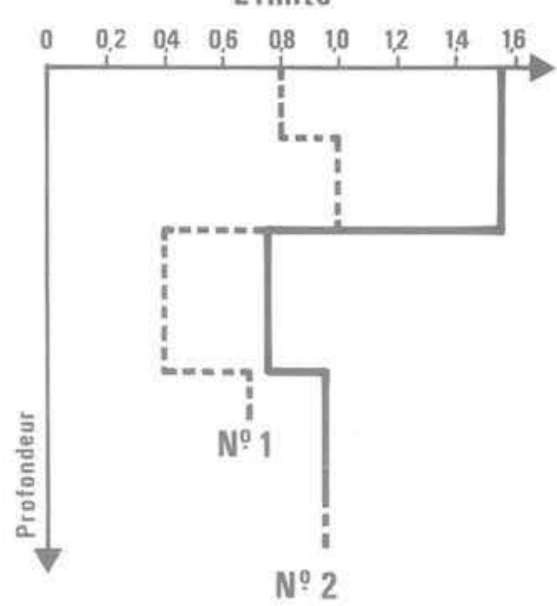

Observations particulières

Bucket cassé sous le pieu $\mathrm{n}^{0} 1$ à $7,5 \mathrm{~m}$

Forage ouvert pendant plus de $24^{\mathrm{h}}$ pour repêchage

Tableau 7 Dunkerque - BCEM (1973-1974)

Type de fondations : pieux métalliques forés et cimentés au coulis par gravité

Géométrie : $₫ 500 \mathrm{~mm}$

Perforation : $\varnothing 660 \mathrm{~mm}$, circulation inverse à l'eau

Profil géotechnique

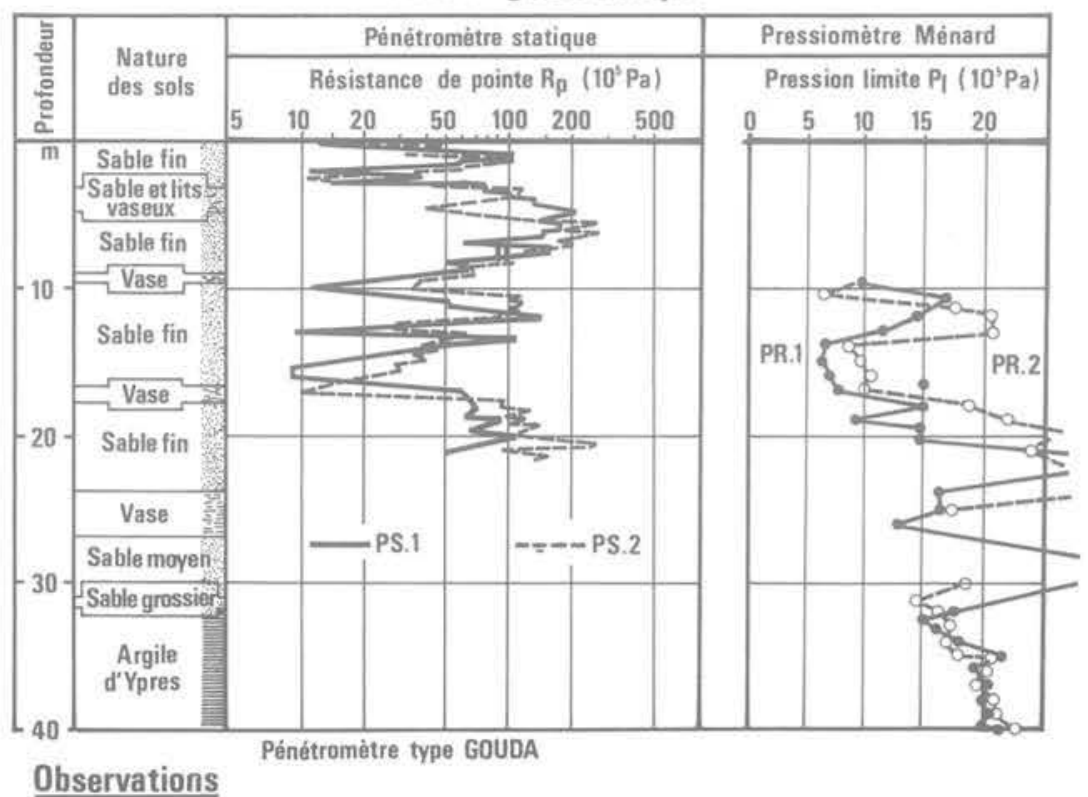

Fiche des pieux

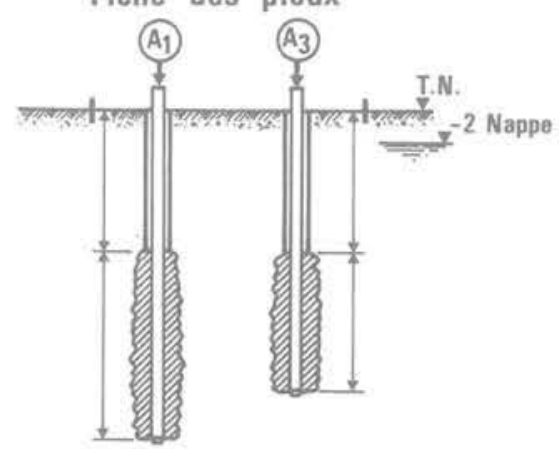

Frottement latéral $f_{\text {lat. }}$ en $10^{5} \mathrm{~Pa}$

\begin{tabular}{|l|l|}
\hline$A_{1}$ & 0,77 \\
\hline$A_{3}$ & 0,36 \\
\hline
\end{tabular}

\section{Observations}

Nombreux éboulements du forage pendant la perforation et la descente du pieu $A_{3}$

Utilisation nécessaire de boue bentonique après l'eau claire 


\section{Effet du délai de repos}

Là encore, il faut distinguer deux possibilités :

- le délai de repos n'entraîne aucune amélioration ou détérioration de la portance (tableaux 8 et 9).

- le facteur temps a une influence sensible sur la portance (ou la capacité d'ancrage) de la fondation. Celle-ci ne cesse de croître, tout au moins pendant une période de temps variable mais toujours limitée (tableau 10).

Cas du tableau 8

Ce tableau présente quelques-uns des résultats obtenus sur le site expérimental de Coudekerque, à la suite du chargement de trois pieux béton, façonnés à l'avance [18]. Ces trois pieux présentent la particularité d'avoir été battus et lancés simultanément sur les premiers mètres, puis uniquement battus pour la mise en fiche des deux derniers mètres dans les sables compacts. En outre, chaque pieu a fait l'objet de deux chargements consécutifs à trois mois d'intervalle. On a pu constater que le frottement latéral unitaire $\left(f_{\text {lat }}\right)$, d'ailleurs nul sur toute la longueur lancée du fût, ne subissait aucune évolution dans le temps au droit des deux derniers metres de fot, uniquement battus.

La permanence de la valeur du frottement latéral $\left(f_{\text {tat }}\right)$ s'explique par la nature du sol environnant (sable moyen perméable) lequel, en raison de sa perméabilité élevée, n'est sujet à aucune évolution de sa résistance au cisaillement.

De même pour les charges dites de fluage $\left(Q_{F}\right)$, le temps n'en a pas fait évoluer les valeurs.

\section{Cas du tableau 9}

Ces résultats constituent le complément du tableau 1 commenté précédemment. II illustre cette fois la permanence du frottement latéral unitaire $\left(f_{\text {lat }}\right)$ au contact du fût du $3^{\circ}$ pieu (métallique), 470 jours après son premier chargement. Le sol remanié ne subit aucune "régénération ». C'est la nature du contact « limon-acier » qui semble avoir joué ici un rôle déterminant, bloquant tout phénomène de reconstitution en raison de l'imperméabilité du fût.

Le cas du tableau 10, comme on va le voir, ne fait que confirmer cette explication.

De la même manière, la résistance limite de pointe $\left(Q_{p}^{L}\right)$ de ce pieu n'a subi aucune augmentation : on retrouvera au $2^{\circ}$ essai la première valeur, soit $1150 \mathrm{kN}$.

\section{Cas du tableau 10}

Ce tableau complète également le tableau 1. II montre l'évolution du frottement latéral unitaire $\left(f_{\text {lat }}\right)$ entre le premier et le deuxième essai pour le deuxième pieu béton, soit après un délai de repos de 135 jours. L'augmentation est sensible d'environ $50 \%$. Au troisième essai cependant, c'est-à-dire à 365 jours du premier, la valeur de $f_{\text {lat }}$ fut égale à celle du deuxième essai. Une augmentation non moins marquée, puisque de l'ordre de $75 \%(0,052 \mathrm{MPa}$ contre $0,090 \mathrm{MPa}$ ) fut également observée, pour le $1^{\text {er }}$ pieu béton, à 304 jours d'intervalle de son premier chargement.

Dans les deux cas, l'amélioration sensible de la qualité du contact « sol-pieu " est à mettre cette fois, au compte du matériau constituant le fût, c'est-à-dire le béton moulé.

Tableau 8

PIEUX: FACONNES A L'AVANCE $(35 \times 35)$

NOMBRE: 3

MISE EN FICHE: $1 \%$ BATTAGE AVEC LANÇAGE SUR PREMIERSM

$2 \%$ BATTAGE POUR LES 2 DERNIERS METRES

EQUIPEMENT: EXTENSOMETRE AMOVIBLE + JAUGES

ESSAIS DE CHARGEMENT: $1 \%$ JUILLET 1973

$2 \%$ OCTOBRE 1973

INFLUENCE DU TEMPS: NULLE

COURBES

D'ENFONCEMENT

PROFIL GEOTECHNIQUE

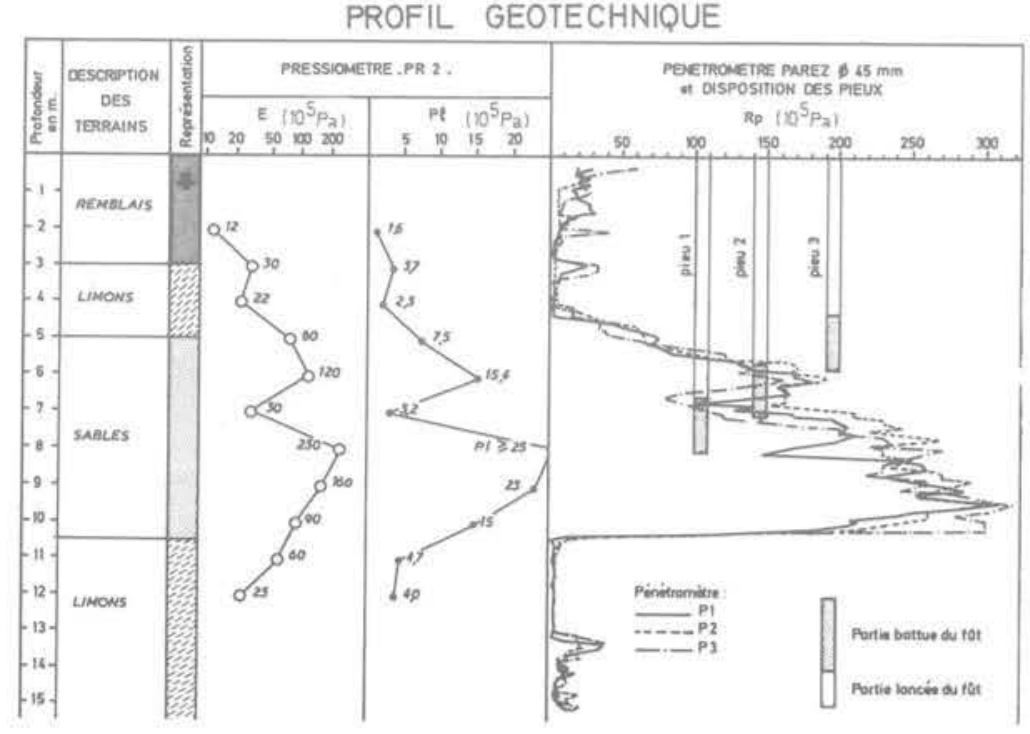

\begin{tabular}{|c|c|c|}
\hline PIEU & $\begin{array}{l}\text { CHARGE DE } \\
\text { FLUAGE } Q_{F} \\
(\mathrm{kN})\end{array}$ & $\begin{array}{l}\text { FROTTEMENT } \\
\text { LATERAL UNITAIRE } \\
\left(10^{5} \mathrm{~Pa}_{\mathrm{a}}\right)\end{array}$ \\
\hline 1 & $\begin{array}{l}1050 \\
(1070)\end{array}$ & $(1,25)$ \\
\hline 2 & $\begin{array}{l}530 \\
(530)\end{array}$ & $\begin{array}{l}0,6 \\
(0,6)\end{array}$ \\
\hline
\end{tabular}

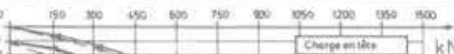

MOBILISATION dU FROTTEMENT LATERAL

(partie battue du pieu)

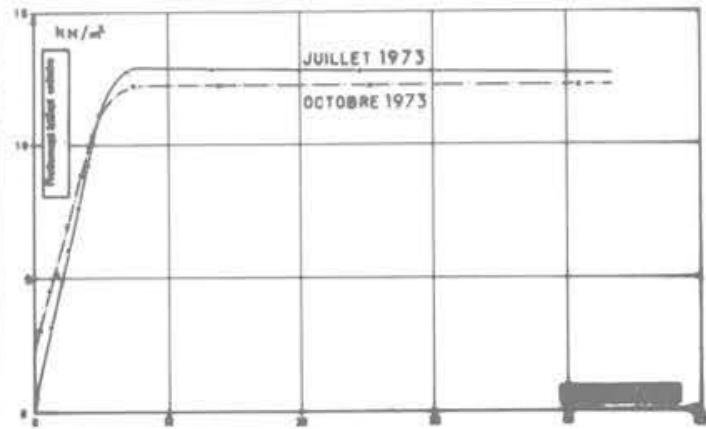


PIEU: TUBULAIRE METALLIOUE VISITABLE $\phi=880 \mathrm{~mm}$ EXECUTION : FORE BENOTO EQUIPEMENT : TELEMAC

ESSAIS DE CHARGEMENT : $1 \% 13$ FEVRIER 1970

INFLUENCE DU TEMPS : NULLE

\begin{tabular}{|c|c|c|c|}
\hline ESSAI & $\begin{array}{c}\text { DELAI } \\
\text { (jours) }\end{array}$ & $\begin{array}{c}\text { CHARGE LIMMTE } \\
\text { EN POINTE } \\
\text { QL (kN) }\end{array}$ & $\begin{array}{c}\text { FROTTEMENT } \\
\text { LATERAL } \\
\text { f lat (1080) }\end{array}$ \\
\hline 1 er & - & 1150 & 0,220 \\
\hline 2 ithe & 470 & 1150 & 0,220 \\
\hline
\end{tabular}

PROFIL GEOTECHNIQUE
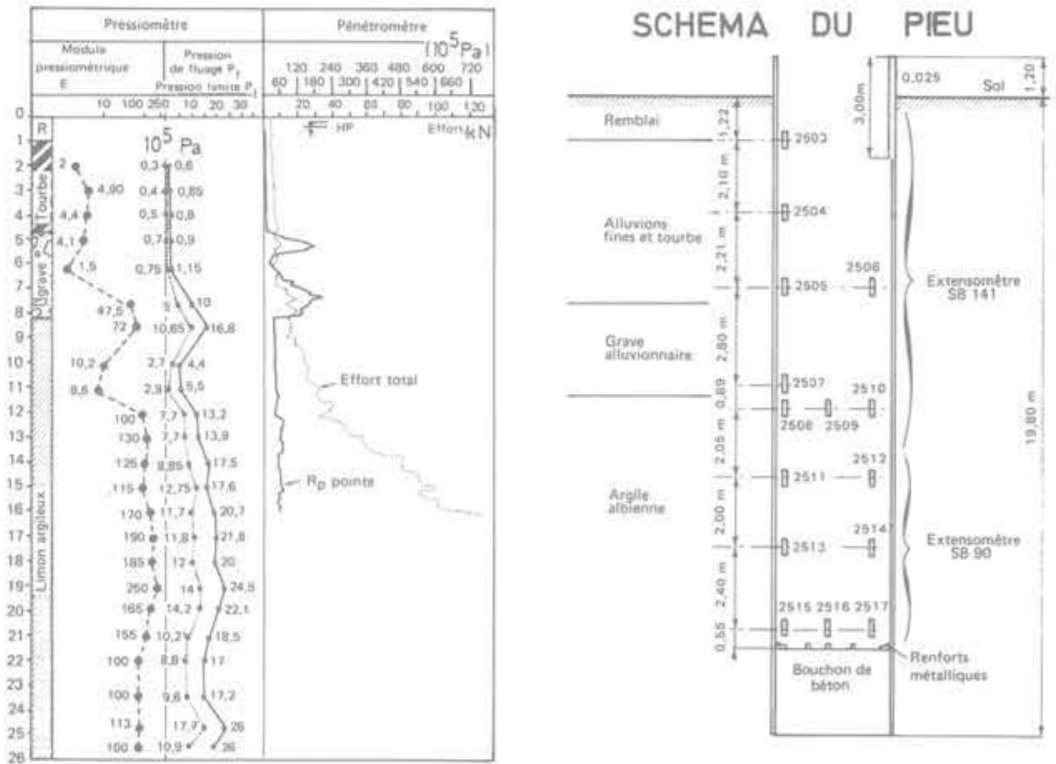

MOBLLISATION

DU FROTTEMENT LATERAL

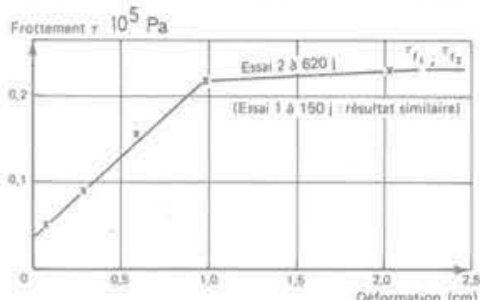

Tableau 10

PIEU : BETON $\sigma=900 \mathrm{~mm}$ (chemisé sur $10 \mathrm{~m}$ )

EXECUTION : FORE BENOTO

EQUIPEMENT : T ELEMAC

ESSAIS DE CHARGEMENT : $1^{\circ} 30$ JANVIER 1970

$2^{\circ} 16-27$ JUIN 1970

$3^{\circ} 29$ JUIN 1970

INFLUENCE DU TEMPS : MARQUEE ENTRE LES

1 er et 2 ème ESSAIS

\begin{tabular}{|c|c|c|c|}
\hline ESSAI & $\begin{array}{l}\text { DELAI } \\
\text { (jours) }\end{array}$ & $\begin{array}{c}\text { CHARGE LIMITE } \\
\text { EN POINTE } \\
Q_{L_{1}}(\mathrm{kN})\end{array}$ & $\begin{array}{l}\text { FROTTEMENT } \\
\text { LATERAL } \\
f_{\text {lot }}\left(10^{5} \mathrm{~Pa}\right)\end{array}$ \\
\hline $1 \mathrm{er}$ & & 1350 & 0,46 \\
\hline 2 ime & 135 & 1500 & 0,68 \\
\hline $3 \mathrm{me}$ & 365 & 1500 & 0,68 \\
\hline
\end{tabular}

MOBILISATION DU FROTTEMENT LATERAL

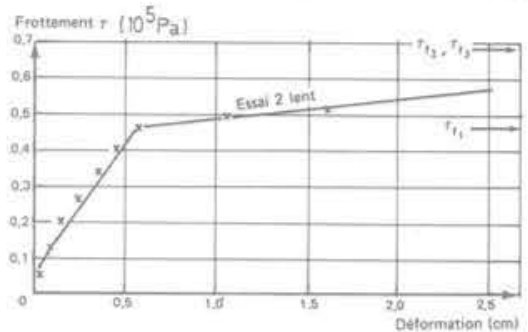

SCHEMA DU PIEU

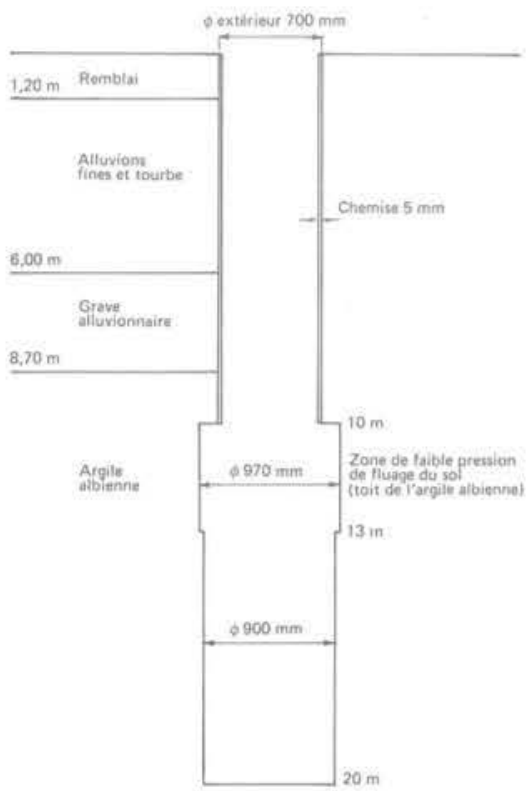

COURBES D'ENFONCEMENT ET DE FLUAGE

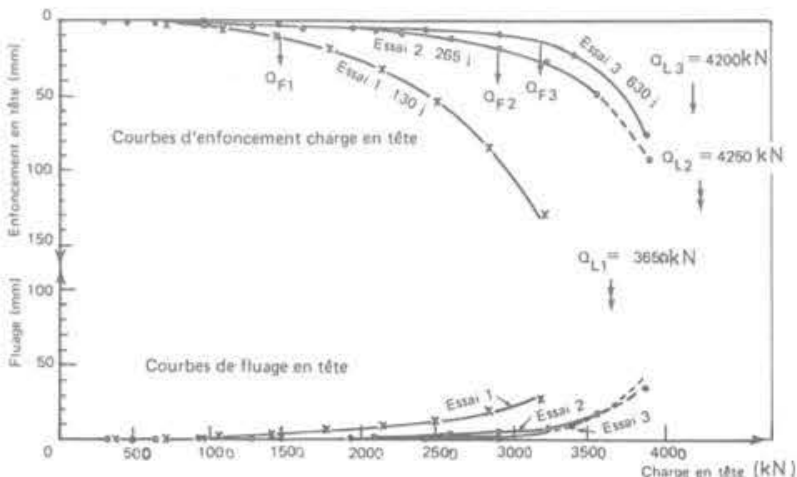




\section{Conclusions}

Si toutes les fondations profondes forées isolées (barrettes, pieux, tirants) obéissent aux mêmes lois de comportement, la prévision de leur capacité portante (ou d'ancrage) reste difficile parce que conditionnée par trois facteurs que l'on ne sait pas intégrer dans les calculs habituels. Ce sont :

- le mode de perforation.

- le délai de repos,

- lincident en cours de forage.

\subsection{Le mode de perforation}

Celui-ci modifie les propriétés du sol, la plupart du temps les altérant.

Dans le cas de sols cohérents particulièrement sensibles et fragiles et donc aptes au remaniement, souvent, il ne sera possible de mobiliser que 30 à $50 \%$ de la résistance au cisaillement du sol intact. Dans les cas les plus défavorables, au plus 10 à $15 \%$. Pour le choix di type de fondation et du mode de perforation dans de pareilles formations, des essais comparatifs de chargement (ou d'arrachement) en vraie grandeur s'avèreront indispensables. On a démontré pour des sols cohérents que le choix judicieux de ces deux éléments conduit à des rapports de portance (ou d'ancrage) pouvant aller de 2 à 4 , voire plus. De tels rapports peuvent aussi être obtenus pour des sols pulvérulents. On a vu que dans ces derniers, certaines techniques de perforation telles que le lancage ou même le battage (cas particulier du milieu frottant légèrement cimenté) peuvent non seulement faire chuter considérablement le frottement latéral mais même l'annuler. II faut souligner enfin, qu'en règle générale, la perforation au coulis de ciment ot toutes les techniques faisant appel à l'injection sous pression ont un effet bénéfique sur la portance (ou l'ancrage) de la fondation.

\subsection{Le délai de repos}

Son effet, qui se fait sentir sur des périodes variables mais toujours limitées, peut être encore une fois considérable pour des sols fins, à condition que la nature du contact «fot-sol » favorise le drainage de ce dernier. C'est ainsi que pour des pieux béton, on a observé des augmentations de frottement latéral unitaire $\left(\mathrm{f}_{\text {lat }}\right)$ de 50 à $75 \%$ de la valeur initiale. Ces gains ont correspondu à des périodes de plusieurs mois.

II semble par contre que le fût métallique bloque tout phénomène de "régénération " d'un sol fin.

De même, pour les sols grossiers et en raison d'ailleurs de leur perméabilité, il ne semble pas que ce phénomène puisse se produire et cela, indépendamment du type de matériau qui constitue le fût (béton ou acier).

Rappelons enfin à ce propos le cas particulier mais bien connu de la craie pour laquelle le délai de repos, en raison d'ailleurs du phénomène de thixotropie, provoque des gains de portance considérables, parfois supérieurs à $100 \%$.

II faut signaler toutefois que pour la craie, lincidence du temps, bien que réelle a un caractère immédiat. c'est-à-dire ne joue que dans les tous premiers jours ou semaines de la mise en place et surtout pour des fondations battues ou vibrofoncées [20].

\subsection{L'incident en cours de forage}

Bien qu'aléatoire, celui-ci a toujours des répercussions défavorables sur la portance (ou l'ancrage) de la fondation. Par la décompression du forage ou le remaniement accru du fond ou des parois, it fait chuter sensiblement le frottement latéral et la résistance de pointe.

Ainsi, pour ce qui est du frottement latéral, on a pu accuser des pertes de $50 \%$ par rapport à des fondations dornt la mise en œuvre s'était déroulée sans incidents. Pour la pointe, en dehors du cas caricatural d'une résistance nulle, due à une malfaçon, de pareils chiffres peuvent être cités.
II est à noter que ces ordres de grandeur ont pu être observés aussi bien pour des sols cohérents que frottants. II faut toutefois faire remarquer qu'à nouveau l'injection de coulis sous pression élevée peut efficacement " reprendre " une fondation " accidentée ». II est courant par exemple de pouvoir restituer par ce biais à la pointe d'un pieu, au moins $50 \%$ de sa résistance initiale.

Néanmoins, il faut garder à l'esprit qu'une fondation dont l'exécution a subi des incidents (éboulements, perte d'outils, forage ouvert trop longtemps, etc.) si elle n'a pas été soigneusement "reprise», accusera presque immanquablement un défaut de portance (ou d'ancrage).

\section{Références Bibliographiques}

[1] MEYERHOFF, - Compaction of sands and bearing capacity of piles. ASCE - proceedings, Déc. 1959. Vol. 85 - SM 6.

[2] JEZEQUEL. - Prévision de la capacité portante du pieu isolé. Conférence de Saint-Quay-Portrieux. Juin 1971, rapport interne.

[3] JEZEQUEL, GUÉGAN et HERVÉ. - Scissomètre rétractable pour l'étude de l'état des sols cohérents autour des pieux. Bull. Liaison des Ponts-et-Chaussées n 56 - Déc. 1971.

[4] BAGUELIN, BUSTAMANTE, FRANCK et JEZEQUEL. - La capacité portante des pieux. Annales de II.T.B.T.P. $n^{\circ} 330$ Juillet-Aout 1975

[5] CAMBEFORT. - Essai sur le comportement en terrain homogène des pieux isolés et des groupes de pieux. Annales de II.T.B.T.P. $n^{\circ}$ 204, Décembre 1964.

[6] BAGUELIN et VENON. - Influence de la compressibilité des pieux sur la mobilisation des efforts résistants. Le comportement des sols avant la rupture. Bulletin Spécial des Laboratoires des Ponts-et-Chaussées, Juin 1972.

[7] Mode opératoire de l'essai statique de fondations profondes LPC. Mai 1970.

[8] Recommandations concernant la conception. le calcul. l'exécution et le controble des tirants d'ancrage. TA-77 Eyrolles 1977.

[9] COMBARIEU. - Essais de chargement de pieux forés dans un limon argileux. Bull. Liaison des Ponts-et-Chaussées $n^{\circ} 80$ Novembre-Décembre 1975 .

[10] KERISEL. - Conclusions du premier cycle de perfectionnement J. VERDEYEN. Institut des constructions civiles, Février 1977. Publications de I'Université Libre de Bruxelles.

[11] KERISEL. - Traité de Mécanique des sols. $4^{\circ}$ édit. p. 395 Éd. Gauthier Villars, 1966

[12] AMERICAN PETROLEUM INSTITUTE. - Recommended practice for fixed Offshore Platforms. 1976

[13] ANGEMEER, CARLSON et KLICK. - Techniques and Results of Offshore Pile Load Testing in. calcareous soils. OTC 1973 page $n^{\circ} 1894$ et OTC 1974 page $n^{\circ} 2311$.

[14] BUSTAMANTE, DELMAS et LACOUR. - Comportement des tirants précontraints dans une argile plastique. Sission spéciale $n^{\circ} 4$. Congrés international de Mécanique des sols. Tokyo 1977. Revue Francaise de Géotechnique $n^{\circ} 3$. Janvier 1978.

[15] BAGUELIN, BUSTAMANTE et JARDIN. - Essais de chargement de pieux forés dans la craie altérée. Bull. Liaison des Ponts-et-Chaussées $n^{\circ} 73$

[16] JARDIN. - Essai statique de chargement d'un pieu dans la craie altérée à Mantes. Rapport de recherche FAER 1.05.02.4 (Publication interne des Laboratoires des Ponts-et-Chaussées. 1975).

[17] BUSTAMANTE, GABAIX et GOUVENOT. - Essais de pieux scellés par injection sous pression. Annales de l'I.T.B.T.P. n 331. Septembre 1975

[18] BUSTAMANTE, DERVAUX et HULO, - Essais de chargement statique de 3 pieux "lancés-battus ». Bulletin de liaison des Laboratoires des Ponts-et-Chaussées $n^{\circ} 84$, Juillet-Août 1976.

[19] PASTUREL - Essais de chargement de pieux dans la craie altirée. Bulletin de liaison des Ponts-et-Chaussées $n^{\circ} 29$. Jarvier-Février 1968 